\title{
Cytokeratin 18 in plasma of patients with gastrointestinal adenocarcinoma as a biomarker of tumour response
}

\author{
LC Scott', TRJ Evans', J Cassidy', S Harden², J Paul'2, R Ullah', V O'Brien³ and R Brown*,3 \\ 'Centre for Oncology and Applied Pharmacology, University of Glasgow, Cancer Research UK Beatson Laboratories, Garscube Estate, Switchback Road, \\ Glasgow G6I IBD, UK; ${ }^{2}$ Cancer Research UK Clinical Trials Unit, Level O, The Beatson West of Scotland Cancer Centre, 1053 Great Western Road, \\ Glasgow GI2 OYN, UK; ${ }^{3}$ Department of Oncology, Cyclotron Building, Hammersmith Hospital, Du Cane Road, London WI 2 ONN, UK
}

BACKGROUND: Plasma biomarkers may be particularly useful as a predictor or early marker of clinical response to treatment in addition to radiological imaging. Cytokeratin $18(C K \mid 8)$ is an epithelial-specific cytokeratin that undergoes cleavage by caspases during apoptosis. Measurement of caspase-cleaved (CKI8-Asp396) or total cytokeratin 18 (CKI8) from epithelial-derived tumours could be a simple, non-invasive way to monitor or predict responses to treatment.

METHODS: Soluble plasma CKI8-Asp396 and CKI8 were measured by ELISA from 73 patients with advanced gastrointestinal adenocarcinomas before treatment and during chemotherapy, as well as 100 healthy volunteers.

RESULTS: Both CKI8-Asp396 and total CKI 8 plasma levels were significantly higher in patients compared with the healthy volunteers $(P=0.015, P<0.001)$. The total $C K I 8$ baseline plasma levels before treatment were significantly higher $(P=0.009)$ in patients who develop progressive disease than those who achieve partial response or stable disease and this correlation was confirmed in an independent validation set. The peak plasma levels of CKI8 occurring in any cycle following treatment were also found to be associated with tumour response, but peak levels of CKI 8 -Asp396 did not reach significance $(P=0.01$, and $P=0.07$, respectively). CONCLUSION: Plasma levels CKI 8 are a potential marker of tumour response in patients with advanced gastrointestinal malignancy. British Journal of Cancer (2009) I 0 I, 4I0-417. doi:10.1038/sj.bjc.6605 I75 www.bjcancer.com

Published online I 4 July 2009

(c) 2009 Cancer Research UK

Keywords: cytokeratin 18; biomarkers; gastrointestinal adenocarcinomas; chemotherapy; apoptosis

Radiological imaging of tumours is an essential part of the practise of oncology, with a crucial role in screening programmes and in the diagnosis and staging of established disease. Furthermore, the assessment of tumour size by imaging, usually with computed tomography $(\mathrm{CT})$ is a key component in determining tumour response in clinical practice. However, the development of a plasma biomarker to monitor treatment response would be advantageous in terms of ease of repeated analysis and use of resources compared with anatomical imaging. In addition, such biomarkers might give an earlier indication of potential response to treatment rather than the time lag, which is necessary to observe changes in tumour size. In patients with malignancy this may avoid administering toxic treatments with little prospect of benefit and hence start alternative treatments earlier.

Biomarkers are defined as characteristics that are objectively measured and evaluated as an indicator of normal biological processes, pathological processes, or pharmacological responses to a therapeutic intervention (Srivastava and Srivastava, 2005). The ideal marker has high sensitivity and specificity for diagnosis; its level should correlate with disease stage and response to treatment; and it should be easily and reproducibly measured. Unfortunately,

*Correspondence: Professor R Brown, Department of Oncology, Cyclotron Building, Hammersmith Hospital Campus, Imperial College, London WI2 ONN, UK; E-mail: b.brown@imperial.ac.uk

Received 9 March 2009; revised 8 June 2009; accepted 9 June 2009; published online 14 July 2009 the biomarkers currently available for use in the management of solid tumours do not fulfil all these criteria and, therefore, are not presently recommended for screening of the general population. At present the main uses of biomarkers are in determining prognosis, monitoring responses to treatment, and in detection of disease recurrence.

Carcinoembryonic antigen (CEA) is a member of a class of oncofetal antigens that are produced within the normal developing fetus, but only in minute amounts by normal adult cells. First described in 1965, it has become the most widely used biomarker in gastrointestinal malignancy (Hostetter et al, 1990) and can be measured quantitatively by an immunoradiometric assay in serum, but owing to its lack of sensitivity in the early stages of disease it is unsuitable for population screening. Although elevated levels were first observed in patients with colorectal cancer, it can also be raised in pancreatic, gastric, lung and breast cancer, and so is not specific to colorectal cancer. It can also be elevated in a number of benign conditions including cirrhosis, inflammatory bowel disease, pancreatitis, and up to $3 \%$ of healthy volunteers.

Studies performed looking at serum biomarkers in gastric adenocarcinoma have failed to provide a biomarker that is sensitive or specific enough for population screening (Byrne et al, 1990; Gaspar et al, 2001; Ucar et al, 2008). Biomarkers that have been studied include CEA, carbohydrate antigen 19-9 (CA19-9) and carbohydrate antigen 72-4 (CA72-4). CA19-9 is a monoclonal antibody raised against a colon carcinoma cell line to detect a monosialoganglioside in patients with gastrointestinal 
cancer (Koprowski et al, 1981). It is elevated in $20-40 \%$ patients with gastric cancer. The CA72-4 assay measures a tumourassociated glycoprotein (TAG-72) using two monoclonal antibodies. Raised serum TAG-72 levels have been observed in $33-59 \%$ patients with gastric cancer (Paterson et al, 1986; Heptner et al, 1989).

An assay that measures circulating soluble cytokeratin 19 (CK19), CYFRA 21-1, is based on two monoclonal antibodies to CK19 (Bodenmuller et al, 1994). CYFRA 21-1 has mostly been used clinically in lung, and head and neck cancers (Nisman et al, 1998; Yen et al, 1998). In patients with oesophageal cancer high preoperative levels of CYFRA 21-1 have been found to be associated with tumour progression and poor survival outcomes especially in patients with squamous cell carcinoma (Brockmann et al, 2000; Shimada et al, 2003). Serum squamous cell carcinoma antigen 2 (SCC-antigen) mRNA concentrations have been found to be associated with pathological changes in oesophageal cancer, but are not sensitive or specific enough to be used for screening (Yang et al, 2008).

At present CEA can be used to monitor treatment response in colorectal cancer in patients with elevated levels at baseline. However, there is no such biomarker available for the management of gastro-oesophageal cancer and it would be valuable to have a generic biomarker that could be applicable in routine clinical practise in monitoring treatment response in patients with both upper and lower gastrointestinal tract cancers receiving a range of different chemotherapy regimens.

Cytokeratin 18 (CK18) is a major component of the intermediate filament of simple epithelial cells and epithelial-derived tumours, and makes up approximately $5 \%$ of the total cell protein (Chou et al, 1993). It undergoes cleavage by caspases 3, 7, and 9 during apoptosis into proteolytic fragments (Caulin et al, 1997; Ku et al, 1997; MacFarlane et al, 2000). The monoclonal antibody, M30, recognises a neo-epitope of CK18 (CK18-Asp396 cleavage product) exposed after caspase-mediated cleavage during apoptosis, but not intact CK18 (Leers et al, 1999). A further ELISA (M65) uses two monoclonal antibodies specific for epitopes on CK18 to measure total (both caspase-cleaved and un-cleaved) soluble CK18. The two ELISAs can be used in conjunction to calculate the relative proportion of caspase-cleaved CK18 to total CK18 in plasma (Biven et al, 2003).

The objectives of this study were to compare plasma levels of caspase-cleaved and total soluble CK18 between healthy volunteers and patients with advanced gastrointestinal adenocarcinomas, and to determine if there was any correlation between changes in plasma cytokeratin 18 levels during palliative chemotherapy and response to treatment, and so determine if measurement of CK18 could potentially be used as a marker of clinical outcome.

We report here for the first time that plasma levels of caspasecleaved and total CK18 are significantly higher in patients with gastrointestinal adenocarcinoma than in healthy volunteers, and that plasma CK18 levels before commencing treatment, and peak levels observed during treatment, may predict response to chemotherapy in patients with gastrointestinal adenocarcinomas.

\section{MATERIALS AND METHODS}

\section{Patients with gastrointestinal adenocarcinomas}

This was a single-centre, prospective, open, non-randomised study at the Beatson Oncology Centre, Glasgow, UK. Eligible patients were those with histologically or cytologically confirmed, locally advanced, or metastatic gastrointestinal adenocarcinoma who were due to start systemic anticancer therapy, with either (a) adenocarcinoma of the colon or rectum receiving fluoropyrimidine-based therapy; or (b) adenocarcinoma of the oesophagus or stomach receiving chemotherapy with a regimen containing a fluoropyrimidine and a platinum analogue. Inclusion criteria also included age $\geqslant 18$ years, ability to comply with study procedures, and life expectancy $>3$ months. Patients who had had systemic anticancer therapy or radiotherapy within the previous 6 weeks were excluded, as were women who were pregnant or lactating. Chemotherapy was administered according to local protocols. Disease response was assessed clinically and by computed tomography (CT) scans performed before commencing chemotherapy and at regular intervals during treatment (after cycles 3 and 6 for patients with gastric or oesophageal cancer and patients with colorectal cancer receiving capecitabine, and after cycles 6 and 12 in patients with colorectal cancer receiving infusional 5-flourouracil-based regimens). Disease response, as defined by the RECIST criteria (James et al, 1999), was determined from the radiology reports for individual patients. Epithelial toxicity experienced by patients during chemotherapy was assessed by review of an individual patient's case records, but detailed toxicity assessments were not prospectively recorded in this study. Sample collection from these patients commenced in November 2004 and is currently still ongoing. The study was approved by the West Glasgow Hospitals Research Ethics Committee, and all patients gave written informed consent before undertaking any studyrelated procedures.

Seventy-three patients with locally advanced or metastatic gastrointestinal adenocarcinomas were recruited in the initial exploratory phase, including 18 patients with oesophageal adenocarcinoma, 19 patients with gastric adenocarcinoma, and 36 patients with adenocarcinoma of the colon or rectum (Table 1).

Table I Table summarising patient demographic data

\begin{tabular}{|c|c|}
\hline Characteristics & Number of patients \\
\hline \multicolumn{2}{|l|}{ Age } \\
\hline $\begin{array}{l}\text { Median }-68 \text { years } \\
\text { Range }-24-88 \text { years }\end{array}$ & 73 \\
\hline \multicolumn{2}{|l|}{ Gender } \\
\hline Male & 41 \\
\hline Female & 32 \\
\hline \multicolumn{2}{|l|}{ Primary tumour site } \\
\hline Colorectal & 36 \\
\hline Oesophageal & 18 \\
\hline Gastric & 19 \\
\hline \multicolumn{2}{|l|}{ Disease extent } \\
\hline Locally advanced & 11 \\
\hline Metastatic & 60 \\
\hline Unknown/adjuvant & 2 \\
\hline \multicolumn{2}{|l|}{ Chemotherapy } \\
\hline Capecitabine & 33 \\
\hline Modified de Gramont & 3 \\
\hline ECF & 32 \\
\hline CF & 3 \\
\hline CarboF & 2 \\
\hline \multicolumn{2}{|l|}{ Chemotherapy cycles } \\
\hline \multicolumn{2}{|l|}{ Median - 4} \\
\hline \multicolumn{2}{|l|}{ Range $-1-12$} \\
\hline \multicolumn{2}{|l|}{ Clinical outcome } \\
\hline Partial response & 16 \\
\hline Stable disease & 25 \\
\hline Disease progression & 27 \\
\hline Non evaluable & 5 \\
\hline
\end{tabular}

$\mathrm{ECF}=$ epirubicin/cisplatin/5-fluorouracil, $\quad \mathrm{CF}=$ cisplatin/5-fluorouracil, $\quad$ CarboF $=$ carboplatin/5-fluorouracil. 
Thirty-two of the patients with gastric or oesophageal adenocarcinoma were treated with combination chemotherapy comprising epirubicin, cisplatin, and 5-FU (ECF), three were treated with a combination of cisplatin and 5-FU (CF), and two with carboplatin and 5-FU (CarboF). Thirty-three of the patients with colorectal cancer were treated with capecitabine monotherapy, and three with infusional 5-FU and folinic acid (modified 'de Gramont' regimen).

Baseline pretreatment plasma samples were collected from all patients undergoing chemotherapy. However, plasma was not available for all time points during treatment, highlighting the difficulties in the collection of clinical samples for this type of study; however, baseline pretreatment plasma samples were collected from all patients undergoing chemotherapy.

\section{Healthy volunteers}

On one occasion, blood samples were collected from 100 healthy volunteers between November 2004 and February 2005. All volunteers completed a short health questionnaire that recorded current health status, previous medical history, family medical history, current medications, and smoking and alcohol intake history. The samples were collected anonymously and volunteers were not followed up subsequently and did not have any further health assessments as part of this study. This study was approved by the West Glasgow Hospitals Research Ethics Committee, and all volunteers gave written informed consent.

\section{Blood collection and preparation}

Twenty milliliters of blood were collected into tubes containing EDTA (Greiner Bio-one Vacuette) before starting chemotherapy (baseline) and before administration of each subsequent course of chemotherapy (day 1) until discontinuation of systemic therapy. Additional $20 \mathrm{ml}$ samples were collected at various time points during chemotherapy courses in selected patients, with the time points dependent on the specific chemotherapy regimen (day 2 for infusional 5-flourouracil regimens in colorectal cancer, and on days 2,8 , and 15 in patients with gastric or oesophageal cancer receiving continuous infusional 5 -flourouracil). The time points for sample collection were chosen to coincide with patients' scheduled appointments for treatment, which depended on the regimen received. Blood samples $(20 \mathrm{ml})$ were collected into tubes containing EDTA (Greiner Bio-one Vacuette) on one occasion from the 100 healthy volunteers.

Plasma was separated within $2 \mathrm{~h}$ of collection by centrifuging the whole blood sample at $1500 \mathrm{~g}$ for $10 \mathrm{~min}$ at $20^{\circ} \mathrm{C}$. The supernatant was then removed, placed in a separate $15 \mathrm{ml}$ falcon tube and centrifuged again using the above conditions. The resulting supernatant was then aliquoted into Eppendorf tubes (Eppendorf AG, Hamburg, Germany) and immediately frozen at $-70^{\circ} \mathrm{C}$ until analysis.

\section{Assay methods}

Samples were assayed in duplicate for CK18-Asp196 using the M30-Apoptosense ELISA (PEVIVA AB, Bromma, Sweden), for which the normal range is $\leqslant 180 \mathrm{Ul}^{-1}$ where 1 unit equals $1.24 \mathrm{pmol}$ (manufacturer's brochure), and for total soluble CK18 using the M65-ELISA (PEVIVA) according to the manufacturer's instructions and the final result used for further analysis was the mean of the values from the duplicates. In brief, $25 \mu \mathrm{l}$ of sample was added to each well, followed by $75 \mu \mathrm{l}$ of HRP-conjugated monoclonal antibody. The samples were then incubated for $4 \mathrm{~h}$ at room temperature with constant shaking, after which excess unbound conjugate was removed by five washing steps. Colour development was then achieved by the addition of $200 \mu \mathrm{l}$ of 3 , $3,5,5^{\prime}$-tetramethyl-benzidine solution, followed by incubation for 20 min in the dark. The reaction was stopped by the addition of
$50 \mu \mathrm{l}$ of $1.0 \mathrm{M}$ sulphuric acid and the absorbance measured in a microplate reader at $450 \mathrm{~nm}$. Through plotting a standard curve of known concentrations of M30 antigen standards supplied in the kit against absorbance, the amount of antigen in the controls and unknown samples can be calculated by interpolation. Sample dilution was performed according to the manufacturer's instructions.

\section{Study design and sample size}

The objectives of this study were to compare plasma levels of CK18-Asp196 and total soluble CK18 between healthy volunteers and patients with advanced gastrointestinal adenocarcinomas, to determine if there was any correlation between changes in plasma cytokeratin 18 levels during palliative chemotherapy and tumour response, and so determine if measurement of CK18 could potentially be used as a marker of clinical outcome. Other variables considered within the patient population included age, gender, disease extent at baseline, tumour marker (CEA) assessment (patient population only), epithelial toxicity and chemotherapy regimen received.

The sample size of 73 cancer patients was initially assessed in an exploratory study as this was the number of patient samples available. As the subsequent statistical analysis yielded positive correlations, a validation study was then performed using an additional 53 patients, a power analysis was performed to ensure that this number was adequate based on the associations observed in the exploratory study.

It was considered inappropriate to perform a survival analysis for this heterogenous group of patients with either advanced gastro-oesophageal or colorectal cancers receiving palliative chemotherapy with a number of chemotherapy regimens. Tumour response was considered a more robust indicator of clinical outcome for the purposes of this study.

\section{Statistical analysis methods}

The distribution of the CK18, CK18-Asp396 and CEA values were markedly skewed so these variables were logged in all analyses. Where there were two classifying groups (e.g., gender) the MannWhitney $U$-test was used to compare these variables (or changes between variable values); when there were more than two groups (e.g., tumour site) the Kruskal-Wallis test was used. Spearman's rank correlation test was used when determining an association between either CK18 or CK18-Asp396 and age. The replicate variability in the assays was estimated from all the replicate results available in the study.

\section{RESULTS}

\section{Total soluble and caspase-cleaved CK18 levels: healthy volunteers}

Plasma CK18 - Asp396 levels within 100 normal healthy volunteers were assessed using the M30 Apoptosense ELISA. Within the population studied there was a wide range of values, from 51 to $849 \mathrm{Ul}^{-1}$, with a median of $121 \mathrm{Ul}^{-1}$. The values were independent of age $(P=0.80)$ and gender $(P=0.21)$. The range in female patients was $51-849 \mathrm{Ul}^{-1}$ and that in male patients was $79-616 \mathrm{Ul}^{-1}$. The total soluble CK18 levels were also determined for the 100 normal healthy volunteers using the M65 ELISA and as for the CK18-Asp396, there was a wide range in total soluble CK18 levels $\left(161-899 \mathrm{Ul}^{-1}\right)$, with a median of $312 \mathrm{Ul}^{-1}$. As for CK18Asp396, the range in females was greater compared to the male patients $\left(167-899\right.$ vs $\left.161-630 \mathrm{Ul}^{-1}\right)$. Again these values were not significantly associated with age $(P=0.45)$ or gender $(P=0.06)$. 
Total soluble and caspase-cleaved CK18: patients with gastrointestinal adenocarcinomas

Baseline CK18-Asp396 and CK18 levels were measured in 73 patients with advanced gastrointestinal adenocarcinoma. The median CK18-Asp396 value was $207 \mathrm{Ul}^{-1}$ (range $35-2535 \mathrm{Ul}^{-1}$ ) and the median CK18 value was $717 \mathrm{Ul}^{-1}$ (range $206-7747 \mathrm{Ul}^{-1}$ ). The pretreatment plasma levels of both CK18-Asp396 and CK18 were significantly higher $(P=0.015$ for patients with gastric cancer and $P<0.001$ for patients with oesophageal and colorectal cancer) in the plasma samples of patients with all types of gastrointestinal adenocarcinoma examined compared with plasma samples from healthy volunteers (Figure 1 and Table 2).

Receiver operating characteristic (ROC) curves for CK18Asp396 and CK18 distinguished between patients with advanced gastrointestinal malignancy and healthy volunteers (Figure 2). Pooled data for all 73 patients demonstrated that CK18-Asp396 has a sensitivity of $27 \%$ at a specificity of $90 \%$ in distinguishing patients with advanced gastrointestinal malignancy and healthy volunteers. Similarly, CK18 has a sensitivity of $71 \%$ at a specificity of $90 \%$ in distinguishing between patients with advanced gastrointestinal malignancy and healthy volunteers. These results suggest that CK18 may be a better biomarker than CK18-Asp396 in distinguishing between plasma from patients with cancer and healthy volunteers, but that both markers may have limited use as a diagnostic marker.

Patients' disease extent at baseline was associated with plasma CK18 and CK18 - Asp396. Both the baseline plasma CK18 - Asp396 and CK18 levels were significantly higher in the patients with metastatic disease compared with those with locally advanced disease; CK18-Asp396, median $210 \mathrm{Ul}^{-1}$ (range 58-2535 $\mathrm{Ul}^{-1}$ ) vs $164 \mathrm{Ul}^{-1}$ (range $35-333 \mathrm{Ul}^{-1}$ ), CK18, median $833 \mathrm{Ul}^{-1}$ (range $\left.260-7747 \mathrm{U} \mathrm{l}^{-1}\right)$ vs $452 \mathrm{Ul}^{-1}$ (range $\left.269-746 \mathrm{Ul}^{-1}\right)(P=0.014$ and $P=0.011$, for CK18-Asp396 and CK18, respectively).

\section{Correlation of plasma CK18 levels with tumour response}

Objective tumour response data was available for 68 out of the 73 patients, and included 16 patients with partial responses, 25 patients with stable disease and 25 patients with progressive disease. A further two patients had rapid clinical disease progression which occurred before radiological disease assessment and were deemed to have progressive disease. Five others were non-evaluable as four had missing scan data and one patient was receiving adjuvant treatment. Patients' case notes were also reviewed to document the timing and grade of epithelial toxicity observed during chemotherapy. This was then correlated with changes in plasma CK18 and CK18Asp396.

The CK18-Asp396 and CK18 median plasma levels at baseline were higher (289 vs $194 \mathrm{Ul}^{-1}$ for CK18-Asp396, and 1021 vs $618 \mathrm{Ul}^{-}$for CK18) in patients who subsequently developed progressive disease during treatment $(n=27)$ compared with patients who subsequently developed partial response or stable disease $(n=41)$, although was only statistically significant for CK18 $(P=0.125, P=0.009$, for CK18-Asp396 and CK18, respectively, see Figure 3 ). There is an overall sensitivity of $22 \%$ at a specificity of $90 \%$ for CK18-Asp396 and an overall sensitivity of $19 \%$ at a specificity of $90 \%$ for CK18 baseline plasma levels in distinguishing between patients who will subsequently progress through chemotherapy and those who will have partial response/ stable disease with treatment.

The plasma levels of CK18-Asp396 and CK18 were examined for each patient and the maximum level (or peak level) observed during treatment, defined as the maximum level that had been observed for each patient during any cycle of treatment, was compared with tumour response. Peak levels of CK18 were found to be associated with tumour response, but peak levels of
A
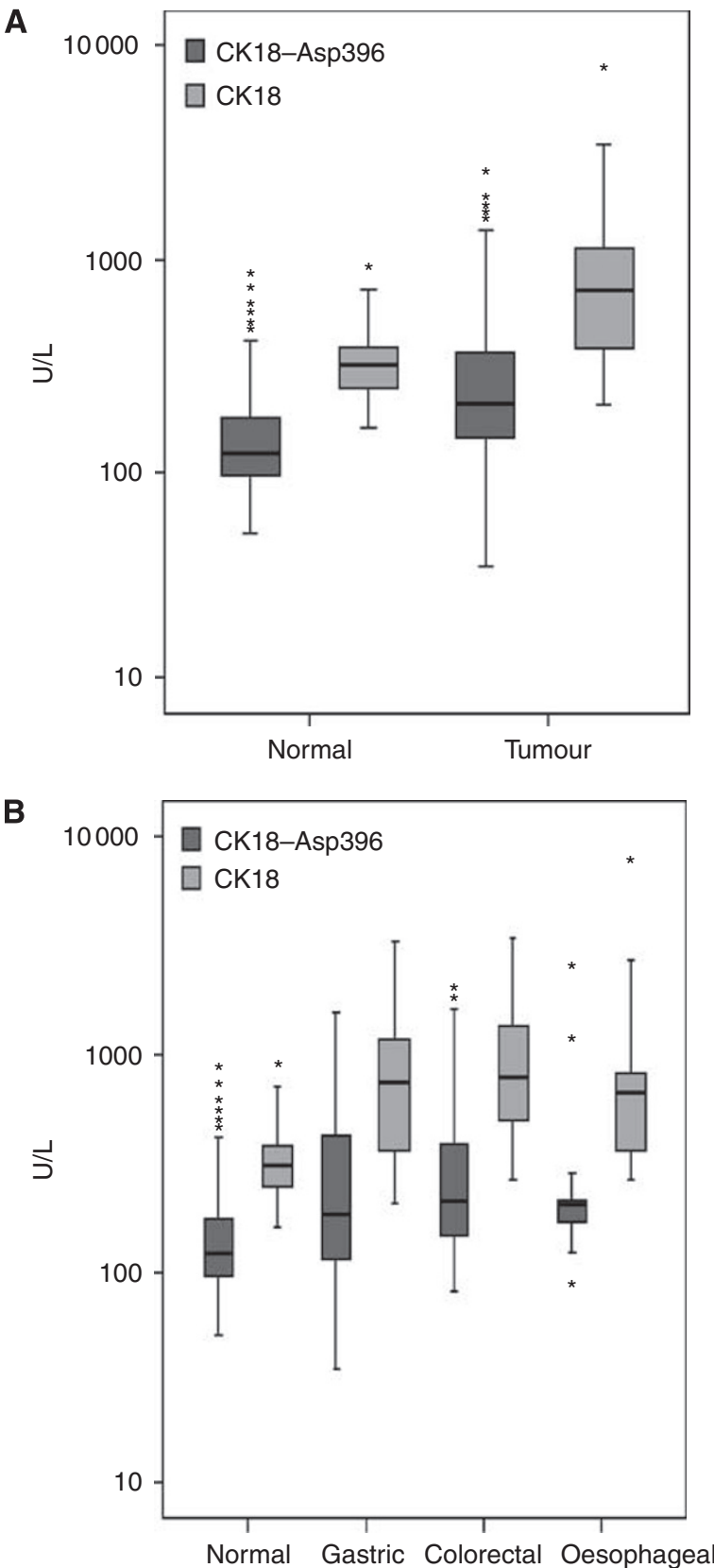

Figure I Plasma CKI8-Asp396 and CKI8 levels in patients vs healthy volunteers, and in different tumour types vs healthy volunteers. (A) Box plot demonstrating significantly higher plasma CKI8-Asp396 and CKI8 levels in patients with advanced gastrointestinal adenocarcinomas compared with healthy volunteers $(P<0.00 \mathrm{I})$. (B) Box plot demonstrating significantly higher baseline CKI8-Asp396 and CKI8 plasma levels in patients with advanced esophageal $(P<0.001$, for CKI8-Asp396 and CKI8), colorectal $(P<0.001$ for CKI8-Asp396 and CKI8) and gastric $(P=0.015, P<0.001$, for CKI8-Asp396 and CKI8, respectively) cancer compared with healthy volunteers. $\mathrm{UI}^{-1}$ is defined according to the manufacturer's brochure where I unit equals 1.24 pmol. Outliers are shown by asterisk.

CK18-Asp396 did not reach significance $(P=0.01$, and $P=0.07$, respectively: Figure $4 \mathrm{~A}$ and $\mathrm{B}$ ).

Comparison of the different chemotherapy regimens (ECF and capecitabine) and tumour response is confounded by comparing responses between the two different tumour groups (upper gastrointestinal cancer and colorectal cancer). Chi-squared test analysis of the relationship between the chemotherapy regimens 
Table 2 Table summarising the median and range of CKI8-Asp396 and CKI8 plasma levels in healthy volunteers and in patients with gastrointestinal adenocarcinoma

\begin{tabular}{|c|c|c|c|c|}
\hline & $\begin{array}{l}\text { CKI } 18-\text { Asp } 396 \text { median } \\
\text { value and range }(U / I)\end{array}$ & $\begin{array}{l}\text { CKI } 8 \text { median value } \\
\text { and range }(U / I)\end{array}$ & $\begin{array}{l}\text { P-value for CKI8-Asp396 between } \\
\text { tumour and healthy volunteers }\end{array}$ & $\begin{array}{l}\text { P-value for CKI 8- between } \\
\text { tumour and healthy volunteers }\end{array}$ \\
\hline Healthy volunteers & $121(51-849)$ & $312(161-899)$ & - & - \\
\hline Gastric cancer & $183(35-1569)$ & $746(206-3313)$ & $P=0.015$ & $P<0.001$ \\
\hline Oesophageal cancer & $204(86-2535)$ & $672(266-7747)$ & $P<0.001$ & $P<0.001$ \\
\hline Colorectal cancer & $213(81-1936)$ & $780(267-3482)$ & $P<0.001$ & $P<0.001$ \\
\hline
\end{tabular}

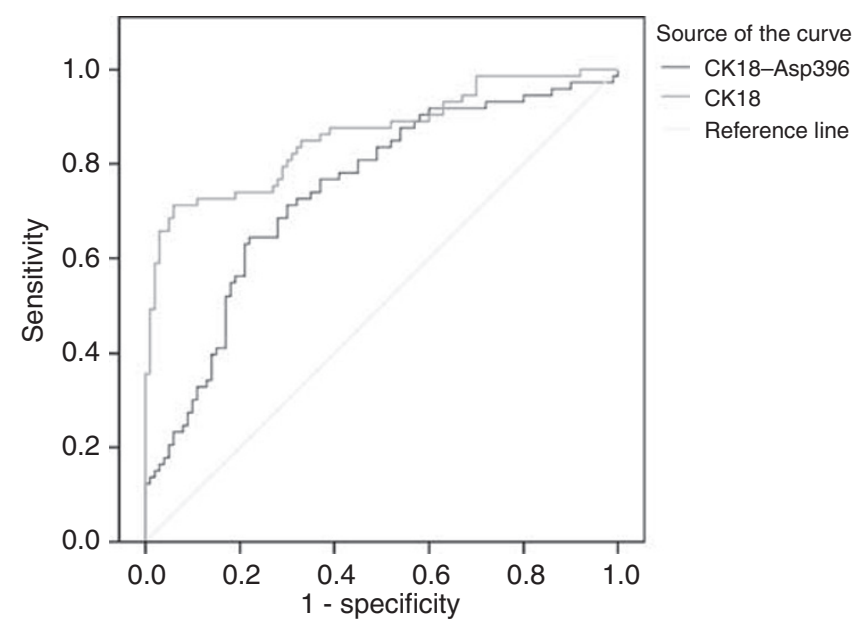

Figure 2 ROC curves for CKI8-Asp396 and CKI8 distinguishing between patients with advanced gastrointestinal malignancy and healthy volunteers. Outliers are shown by asterisk.

and response (partial response/stable disease vs progressive disease) found no relationship between chemotherapy regimen and tumour response. Also, from the analysis the association between baseline plasma CK18-Asp396, total CK18 and response does not appear to be greatly affected when the chemotherapy regimen is allowed for.

Various patient demographic factors, including age, gender, and baseline disease extent (either locally advanced or metastatic disease at commencement of chemotherapy) were then analysed with treatment outcome to chemotherapy, but no correlation was found ( $P=0.514, P=0.149$, and $P=0.934$, for age, gender, and disease extent, respectively). Similarly, there was no significant association between baseline plasma CK18-Asp396 and CK18 levels and patient's age $(P=0.345, P=0.112$ for CK18 - Asp396 and CK18, respectively) and gender $(P=0.519, P=0.257$ for CK18Asp396 and CK18, respectively). In addition, a straightforward visual inspection of the data revealed no obvious association between the timing of epithelial toxicity experienced and the occurrence of peak values of plasma CK18 and CK18-Asp396. However, the sampling for CK18 measurement was within the first 2 days of each cycle of treatment whereas epithelial toxicity is clinically observed mid-way through treatment cycles, although epithelial damage at the cellular level in normal tissues may well occur at the same time as in the cancer but become clinically apparent later during the treatment cycle.

In summary, plasma levels of CK18 at baseline are significantly higher in patients with progressive disease compared to patients with partial response/stable disease, and peak plasma levels of CK18 observed during treatment are associated with treatment response. Differences in plasma CK18-Asp396 and CK18 levels do not significantly associate with the patient's age, gender or the extent of disease at baseline.

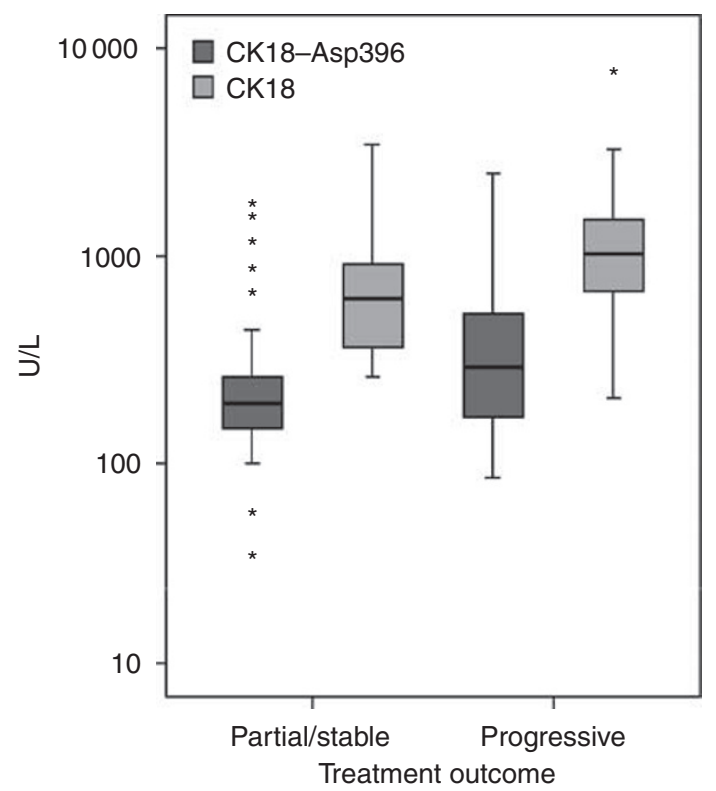

Figure 3 Baseline plasma levels of CKI8-Asp396 and CKI 8 correlated with treatment outcome. Box plot demonstrating baseline CKI8-Asp396 and CKI 8 plasma levels in patients who developed progressive disease through chemotherapy and partial response/stable disease. The CKI 8 level is significantly higher in patients with progressive disease $(P=0.009$, MannWhitney), (NE stands for non-evaluable treatment outcome). $\mathrm{UI}^{-1}$ is defined according to the manufacturer's brochure where | unit equals I.24 pmol. Outliers are shown by asterisk.

\section{Validation study}

A validation study was performed to see if baseline plasma levels of CK18-Asp396 and CK18 showed a reproducible correlation with treatment response in an additional cohort of 53 patients with advanced gastrointestinal malignancy. This included 25 patients with colorectal cancer, 15 with gastric cancer, and 13 with oesophageal cancer. Twenty-five of the patients with gastric or oesophageal adenocarcinoma were treated with combination chemotherapy comprising epirubicin, cisplatin, and 5-FU (ECF), one was treated with a combination of cisplatin and 5-FU (CF), one with carboplatin and 5-FU (CarboF) and one with epirubicin, carboplatin, and 5-FU (ECarboF). Fifteen of the patients with colorectal cancer were treated with capecitabine monotherapy, two with infusional 5-FU and folinic acid (modified 'de Gramont' regimen), five with combination chemotherapy comprising capecitabine and oxaliplatin, one with oxaliplatin and 5-FU (FOLFOX) and two with capecitabine, oxaliplatin, and cetuximab (see Table 3).

Objective tumour response data was available for all of the 53 patients, and included eight patients with partial responses, 24 patients with stable disease and 12 patients with progressive disease. A further nine patients had rapid clinical disease 
Cytokeratin I8: a biomarker in gastrointestinal adenocarcinoma LC Scott et al

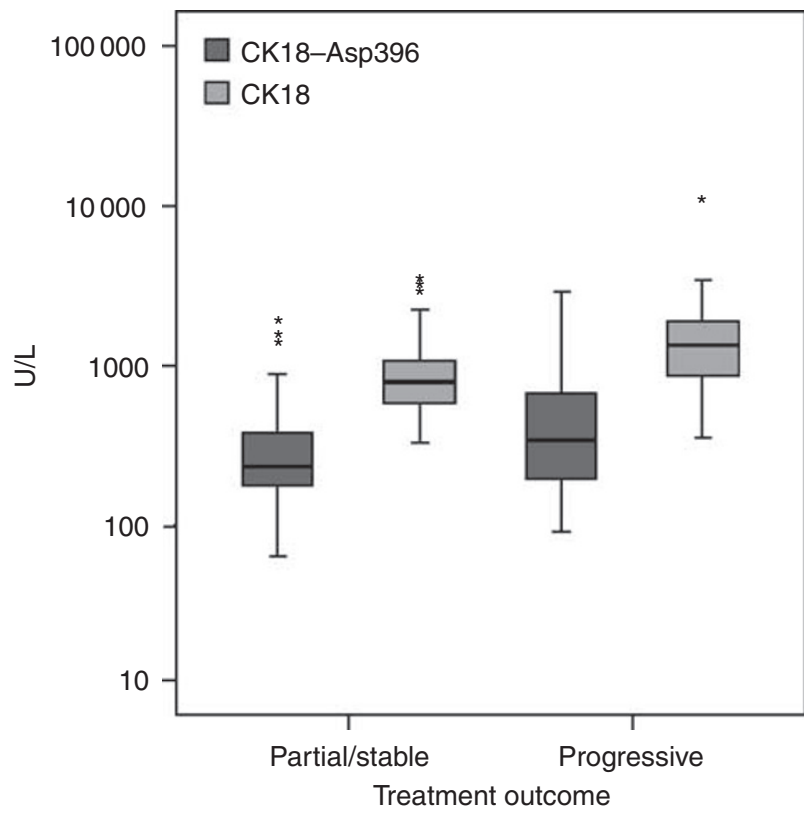

Figure 4 Peak plasma levels of CKI8-Asp396 and CKI8 correlated with clinical response (partial response/stable disease vs progressive disease). Box plot demonstrating peak plasma levels of CKI8-Asp396 and $\mathrm{CKI} 8$ in patients who developed progressive disease through chemotherapy compared with those who achieved a partial response/ stable disease. The peak CKI 8 level is significantly with response $(P=0.0 \mathrm{I})$. Outliers are shown by asterisk.

progression, which occurred before radiological disease assessment and were deemed to have progressive disease.

The validation group showed overlapping range and similar median values for CK18-Asp396 and CK18 as the original test set (median $158 \mathrm{Ul}^{-1}$, range $56-18786 \mathrm{Ul}^{-1}$ for CK18-Asp396 and median $660 \mathrm{Ul}^{-1}$ and range $235-19702 \mathrm{Ul}^{-1}$ for CK18). In the validation group, baseline plasma levels of both CK18-Asp396 and CK18 were again significantly associated with treatment outcomes (partial response/stable disease $v s$ disease progression: $P=0.028$, $P=0.003$, respectively).

\section{DISCUSSION}

This is the first report, to our knowledge, documenting that the mean plasma pretreatment CK18 level is higher in patients with advanced gastrointestinal malignancy compared with healthy volunteers (Figure 1, Table 2). The groups were not age-matched, but levels of CK18 do not correlate with age. It is of note that the range of both plasma CK18-Asp396 and CK18 was wide in the healthy volunteers (Figure 1). Alcohol intake is known to increase caspase-cleaved CK18 values in serum as alcohol may cause apoptosis of liver cells (Natori et al, 2001). Other studies have also shown that viral illness, chronic hepatitis, and sepsis will increase levels of caspase-cleaved CK18 detected by the M30 Apoptosense ELISA kit (Bantel et al, 2004; Roth et al, 2004; Kronenberger et al, 2005). Thus the wide variation in caspase-cleaved CK18 values in healthy volunteers could possibly be due to intercurrent viral illness or alcohol consumption; however, the entry criteria for the healthy volunteer part of the study stated that they should have no significant past medical history, no evidence of intercurrent illness and no concomitant medications. A Mann-Whitney test was used to establish if an alcohol consumption of $<$ or $>10$ units per week affected the plasma CK18 levels in the healthy volunteers. The cutoff of $10 \mathrm{U}$ per week was selected as this is below the recommended weekly consumption for both men and women.
Table 3 Table summarising the patient demographic data in the validation study

\begin{tabular}{|c|c|}
\hline Characteristics & Number of patients \\
\hline \multicolumn{2}{|l|}{ Age } \\
\hline \multicolumn{2}{|l|}{ Median 70 years } \\
\hline \multicolumn{2}{|l|}{ Range $41-86$ years } \\
\hline \multicolumn{2}{|l|}{ Gender } \\
\hline Male & 31 \\
\hline Female & 22 \\
\hline \multicolumn{2}{|l|}{ Primary tumour site } \\
\hline Colorectal & 25 \\
\hline Oesophageal & 13 \\
\hline Gastric & 15 \\
\hline \multicolumn{2}{|l|}{ Disease extent } \\
\hline Locally advanced & 14 \\
\hline Metastatic & 39 \\
\hline \multicolumn{2}{|l|}{ Chemotherapy } \\
\hline Capecitabine & 15 \\
\hline Modified de Gramont & 2 \\
\hline XELOX+1-cetuximab & 2 \\
\hline FOLFOX & I \\
\hline ECF & 25 \\
\hline CF & 1 \\
\hline Carbo F & 1 \\
\hline ECarboF & 1 \\
\hline \multicolumn{2}{|l|}{ Chemotherapy cycles } \\
\hline \multicolumn{2}{|l|}{ Median 2} \\
\hline \multicolumn{2}{|l|}{ Range $1-12$} \\
\hline \multicolumn{2}{|l|}{ Clinical outcome } \\
\hline Partial response & 8 \\
\hline Stable disease & 24 \\
\hline Disease progression & 21 \\
\hline
\end{tabular}

XELOX = oxaliplatin/capecitabine; FOLFOX = oxaliplatin/infusional 5 -flourouracil; $E C F=$ epirubicin/cisplatin/5-flourouracil; $\quad C F=$ cisplatin/5-flourouracil; $\quad$ CarboF $=$ carboplatin/5-flourouracil; EcarboF = epirubicin/carboplatin/5-flourouracil.

Within this group, alcohol consumption was not found to be associated with elevated plasma levels of either CK18-Asp396 or total soluble CK18 ( $P=0.91$ and $P=0.98$, respectively).

The group of healthy volunteers was not followed up long-term and so it is unknown whether they subsequently developed any pathology to account for the variation in levels observed. As there is a significant overlap in plasma CK18-Asp396 levels between the healthy volunteers and cancer patients, it may be challenging to draw conclusions in individual cases.

After our sample collection from healthy volunteers had been completed, it was reported that elevated serum levels of caspasecleaved CK18 may be an indicator of myocardial damage (Adlbrecht et al, 2007). However, healthy volunteers were ineligible if they had significant past or current illnesses. Although it was feasible that the patients with gastrointestinal cancer could have developed myocardial damage during the course of the study, these chemotherapy regimens are used with caution in patients with significant cardiac co-morbidities and we believe that it is unlikely that myocardial damage accounts for the levels of plasma CK18 observed.

We report in this study that the plasma CK18 level before commencing chemotherapy may predict treatment outcome in patients with advanced gastrointestinal adenocarcinoma. The patients with comparatively higher baseline levels of plasma CK18 tended to have higher levels of disease progression through chemotherapy compared to patients with lower baseline levels 
(Figure 3). This may be a reflection of the extent of disease present and potential for access to the circulatory system, as patients with metastatic disease had higher baseline levels of CK18 and CK18Asp396 than those with locally advanced disease. However, when baseline disease extent as determined by CT scanning was correlated with treatment outcome, a statistically significant association was not found. This suggests that the baseline plasma CK18-Asp396 and CK18 levels may give an indication of both tumour burden and also the amount of cell death that is occurring, whether this is as a result of chemotherapy or as part of the ongoing disease process.

Pretreatment levels of CK18 - Asp396 in the sera of patients with primary breast cancer, $(n=152)$, recurrent breast cancer $(n=49)$ or normal controls $(n=82)$ (Ueno et al, 2003), demonstrated that patients with primary cancer had higher serum CK18-Asp396 levels than the normal controls $(P=0.0001)$ and that patients with recurrent cancer had higher serum CK18-Asp396 levels than both the primary breast cancer patients and the normal controls (median values 180.5 vs 165.7 vs $156.2 \mathrm{Ul}^{-1} \quad(P<0.0001$ and $P=0.008$, respectively)). In addition, in patients with recurrent cancer the serum CK18-Asp396 level correlated with the number of involved organs, or burden of disease $(P=0.041)$. In another study in patients with recurrent breast cancer $(n=32)$ receiving chemotherapy with cyclophosphamide, epirubicin and 5-flourouracil or docetaxel (Biven et al, 2003), an index was calculated for each patient based on the difference between the maximum CK18Asp396 level observed during treatment and the pretreatment level. Increases in serum CK18-Asp396 of at least 50\% were significantly associated with clinical response $(P=0.0001)$. More recently, the use of $\mathrm{CK} 18$ as a biomarker for monitoring chemotherapy-induced cell death in breast cancer has been published (Olofsson et al, 2007). In this study, both CK18Asp396 and total CK18 were assessed using the ELISAs and druginduced release of CK18 examined from breast carcinoma cells and tissue. Serum CK18 levels were then determined in 61 patients with breast cancer receiving either docetaxel or cyclophosphamide/ epirubicin/5-flourouracil (CEF) chemotherapy. The results showed that CK18-Asp396 was released from cell and tissue cultures to the extracellular compartment and that the protein complexes were stable, suggesting that CK18-based assays would be applicable in clinical studies. In patients with breast cancer, docetaxel was found to induce increased levels of CK18-Asp396, indicating that the primary mode of cell death was apoptosis. In contrast, CEF induced increased levels of total CK18, indicating that the primary mode of cell death in these patients was necrosis. Also, the level of increase of total $\mathrm{CK} 18$ at $24 \mathrm{hrs}$ post-treatment correlated with clinical response to CEF $(P<0.0001)$. Recently published studies of patients with hormone refractory prostate cancer $(n=82)$ receiving palliative chemotherapy (Kramer et al, 2006) show significant increases in CK18-Asp396 usually between days 5 and 7 of each treatment cycle.

Two studies were recently published evaluating CK18 in patients with colorectal cancer. The first studied pre- and post-operative serum levels of CK18-Asp396 in 31 patients (Ausch et al, 2009). It also assessed serum levels of CK18-Asp396 in 10 patients receiving combination chemotherapy with oxaliplatin/capecitabine. The results showed that peri-operative levels of CK18-Asp 396 correlated significantly with tumour recurrence $(P=0.016)$, but that increases in CK18-Asp 396 observed during chemotherapy did not correlate with response. The second study measured pre- and post-operative plasma levels of CK18-Asp396 and total CK18 in 49 patients with colorectal cancer and correlated the levels with patient and tumour characteristics, and survival outcomes (Koelink et al, 2009). The results showed that peri-operative plasma levels of both CK18-Asp396 and total CK18 were correlated with disease stage and were predictive of disease-free survival independent of tumour stage. Also the ratio of plasma CK18-Asp396/total CK18 which decreased with tumour progression, was also predictive of disease-free survival.

CK18-Asp 396 may also be applicable as a pharmacodynamic biomarker in phase I clinical trials of novel non-cytotoxic molecularly targeted anticancer therapies, with which objective tumour responses, as determined by a reduction in tumour dimensions by conventional imaging techniques, may not be observed. For example, our group has previously demonstrated that disease stabilisation was associated with CK18-Asp 396 plasma levels in patients with advanced solid tumours treated in a phase 1 clinical trial of the novel hydroxamate histone deacetylase inhibitor, belinostat (Steele et al, 2008).

It is of note that since our study started several papers have been published regarding sample-handling protocols for determination of serum and plasma CK18. Delays of greater than $4 \mathrm{~h}$ in sample acquisition and processing resulted in a significant increase in CK18-Asp396 levels. This effect was minimised by incubating the sample on ice. Furthermore, prolonged storage ( $>6$ months) at $-80^{\circ} \mathrm{C}$ resulted in higher levels of CK18-Asp396. The recommendation was that serum rather than plasma should be used to decrease the variation between the duplicate assays (Greystoke et al, 2008). Although plasma was used in our study, we had a robust standard operating protocol for sample acquisition that ensured all samples were processed and frozen to $-80^{\circ} \mathrm{C}$ within $2 \mathrm{~h}$ of venesection. Other studies published have reported the stability of both CK18-Asp396 and total soluble CK18 in plasma from cancer patients stored at $-80^{\circ} \mathrm{C}$ for over 2 years (Cummings et al, 2007). The plasma used in our study had been kept at $-80^{\circ} \mathrm{C}$ for $<2$ years and care was taken to use samples that had not been repeatedly freeze thawed.

In conclusion, the results from our study suggest that measuring baseline and peak plasma levels of CK18 in patients receiving palliative chemotherapy for advanced gastrointestinal malignancy may help predict individual outcomes to therapy. However, a larger prospective clinical study is required to validate these results.

\section{ACKNOWLEDGEMENTS}

This study was supported by Cancer Research UK clinical research fellow Grant (ref C973/A6780), programme Grants (ref C536/ A6689, C973/A4284) and ECMC Grant (ref C973/A7281).

\section{REFERENCES}

Adlbrecht C, Hoetzenecker K, Posch M, Steiner S, Kopp C, Hacker S, Auer J, Horvath R, Moser B, Roth G, Wolner E, Lang IM, Ankersmit HJ (2007) Elevated levels of interleukin-1beta-converting enzyme and caspasecleaved cytokeratin-18 in acute myocardial infarction. Eur J Clin Invest 37: $372-380$

Ausch C, Buxhofer-Ausch V, Olszewski U, Hinterberger W, Ogris E, Schiessel R, Hamilton G (2009) Caspase-cleaved cytokeratin 18 fragment (M30) as marker of postoperative residual tumor load in colon cancer patients. Eur J Surg Oncol (in press)
Bantel H, Lugering A, Heidemann J, Volkmann X, Poremba C, Strassburg CP, Manns MP, Schulze-Osthoff K (2004) Detection of apoptotic caspase activation in sera from patients with chronic $\mathrm{HCV}$ infection is associated with fibrotic liver injury. Hepatology 40: $1078-1087$

Biven K, Erdal H, Hagg M, Ueno T, Zhou R, Lynch M, Rowley B, Wood J, Zhang C, Toi M, Shoshan MC, Linder S (2003) A novel assay for discovery and characterization of pro-apoptotic drugs and for monitoring apoptosis in patient sera. Apoptosis 8: 263-268 
Bodenmuller H, Donie F, Kaufmann M, Banauch D (1994) The tumor markers TPA, TPS, TPACYK and CYFRA 21-1 react differently with the keratins 8, 18 and 19. Int J Biol Markers 9: 70-74

Brockmann JG, St Nottberg H, Glodny B, Sprakel B, Senninger N (2000) Analysis of serum CYFRA 21-1 concentrations in patients with esophageal cancer. Anticancer Res 20: 4899-4904

Byrne DJ, Browning MC, Cuschieri A (1990) CA72-4: a new tumour marker for gastric cancer. Br J Surg 77: $1010-1013$

Caulin C, Salvesen GS, Oshima RG (1997) Caspase cleavage of keratin 18 and reorganization of intermediate filaments during epithelial cell apoptosis. J Cell Biol 138: $1379-1394$

Chou CF, Riopel CL, Rott LS, Omary MB (1993) A significant soluble keratin fraction in 'simple' epithelial cells. Lack of an apparent phosphorylation and glycosylation role in keratin solubility. J Cell Sci 105(Part 2): $433-444$

Cummings J, Ranson M, Butt F, Moore D, Dive C (2007) Qualification of M30 and M65 ELISAs as surrogate biomarkers of cell death: long term antigen stability in cancer patient plasma. Cancer Chemother Pharmacol 60: $921-924$

Gaspar MJ, Arribas I, Coca MC, Diez-Alonso M (2001) Prognostic value of carcinoembryonic antigen, CA 19-9 and CA 72-4 in gastric carcinoma. Tumour Biol 22: 318-322

Greystoke A, Cummings J, Ward T, Simpson K, Renehan A, Butt F, Moore D, Gietema J, Blackhall F, Ranson M, Hughes A, Dive C (2008) Optimisation of circulating biomarkers of cell death for routine clinical use. Ann Oncol 19: 990 - 995

Heptner G, Domschke S, Domschke W (1989) Comparison of CA 72-4 with CA 19-9 and carcinoembryonic antigen in the serodiagnostics of gastrointestinal malignancies. Scand J Gastroenterol 24: $745-750$

Hostetter RB, Augustus LB, Mankarious R, Chi KF, Fan D, Toth C, Thomas P, Jessup JM (1990) Carcinoembryonic antigen as a selective enhancer of colorectal cancer metastasis. J Natl Cancer Inst 82: $380-385$

James K, Eisenhauer E, Christian M, Terenziani M, Vena D, Muldal A, Therasse P (1999) Measuring response in solid tumors: unidimensional vs bidimensional measurement. J Natl Cancer Inst 91: 523 -528

Koelink PJ, Lamers CB, Hommes DW, Verspaget HW (2009) Circulating cell death products predict clinical outcome of colorectal cancer patients. BMC Cancer 9: 88

Koprowski H, Herlyn M, Steplewski Z, Sears HF (1981) Specific antigen in serum of patients with colon carcinoma. Science 212: $53-55$

Kramer G, Schwarz S, Hagg M, Havelka AM, Linder S (2006) Docetaxel induces apoptosis in hormone refractory prostate carcinomas during multiple treatment cycles. Br J Cancer 94: 1592 - 1598

Kronenberger B, Wagner M, Herrmann E, Mihm U, Piiper A, Sarrazin C, Zeuzem S (2005) Apoptotic cytokeratin 18 neoepitopes in serum of patients with chronic hepatitis C. J Viral Hepat 12: $307-314$

$\mathrm{Ku}$ NO, Liao J, Omary MB (1997) Apoptosis generates stable fragments of human type I keratins. J Biol Chem 272: 33197-33203

Leers MP, Kolgen W, Bjorklund V, Bergman T, Tribbick G, Persson B, Bjorklund P, Ramaekers FC, Bjorklund B, Nap M, Jornvall H, Schutte B
(1999) Immunocytochemical detection and mapping of a cytokeratin 18 neo-epitope exposed during early apoptosis. J Pathol 187: 567-572

MacFarlane M, Merrison W, Dinsdale D, Cohen GM (2000) Active caspases and cleaved cytokeratins are sequestered into cytoplasmic inclusions in TRAIL-induced apoptosis. J Cell Biol 148: 1239-1254

Natori S, Rust C, Stadheim LM, Srinivasan A, Burgart LJ, Gores GJ (2001) Hepatocyte apoptosis is a pathologic feature of human alcoholic hepatitis. J Hepatol 34: 248-253

Nisman B, Lafair J, Heching N, Lyass O, Baras M, Peretz T, Barak V (1998) Evaluation of tissue polypeptide specific antigen, CYFRA 21-1, and carcinoembryonic antigen in nonsmall cell lung carcinoma: does the combined use of cytokeratin markers give any additional information? Cancer 82: 1850 - 1859

Olofsson MH, Ueno T, Pan Y, Xu R, Cai F, van der Kuip H, Muerdter TE, Sonnenberg M, Aulitzky WE, Schwarz S, Andersson E, Shoshan MC, Havelka AM, Toi M, Linder S (2007) Cytokeratin-18 is a useful serum biomarker for early determination of response of breast carcinomas to chemotherapy. Clin Cancer Res 13: 3198-3206

Paterson AJ, Schlom J, Sears HF, Bennett J, Colcher D (1986) A radioimmunoassay for the detection of a human tumor-associated glycoprotein (TAG-72) using monoclonal antibody B72.3. Int J Cancer 37: $659-666$

Roth GA, Krenn C, Brunner M, Moser B, Ploder M, Spittler A, Pelinka L, Sautner T, Wolner E, Boltz-Nitulescu G, Ankersmit HJ (2004) Elevated serum levels of epithelial cell apoptosis-specific cytokeratin 18 neoepitope $\mathrm{m} 30$ in critically ill patients. Shock 22: $218-220$

Shimada H, Nabeya Y, Okazumi S, Matsubara H, Miyazawa Y, Shiratori T, Hayashi H, Gunji Y, Ochiai T (2003) Prognostic significance of CYFRA 21-1 in patients with esophageal squamous cell carcinoma. J Am Coll Surg 196: $573-578$

Srivastava S, Srivastava RG (2005) Proteomics in the forefront of cancer biomarker discovery. J Proteome Res 4: 1098-1103

Steele NL, Plumb JA, Vidal L, Tjornelund J, Knoblauch P, Rasmussen A, Ooi CE, Buhl-Jensen P, Brown R, Evans TR, DeBono JS (2008) A phase 1 pharmacokinetic and pharmacodynamic study of the histone deacetylase inhibitor belinostat in patients with advanced solid tumors. Clin Cancer Res 14: $804-810$

Ucar E, Semerci E, Ustun H, Yetim T, Huzmeli C, Gullu M (2008) Prognostic value of preoperative CEA, CA 19-9, CA 72-4, and AFP levels in gastric cancer. Adv Ther 25: $1075-1084$

Ueno T, Toi M, Biven K, Bando H, Ogawa T, Linder S (2003) Measurement of an apoptotic product in the sera of breast cancer patients. Eur J Cancer 39: $769-774$

Yang YF, Li H, Xu XQ, Diao YT, Fang XQ, Wang Y, Zhao DL, Wu K, Li HQ (2008) An expression of squamous cell carcinoma antigen 2 in peripheral blood within the different stages of esophageal carcinogenesis. Dis Esophagus 21: 395-401

Yen TC, Lin WY, Kao CH, Cheng KY, Wang SJ (1998) A study of a new tumour marker, CYFRA 21-1, in squamous cell carcinoma of the head and neck, and comparison with squamous cell carcinoma antigen. Clin Otolaryngol Allied Sci 23: 82-86 